\title{
The Benefits of Expressed Maternal Milk and Donor Breast Milk for Preventing Necrotizing Enterocolitis in Preterm Infants: Systematic Review and Meta- Analysis
}

\author{
Xiao-Ming Ben*, Rui Chen, Zong-Tai Feng, Tao-ying Chen and Jin-Han Zhang
}

Department of Neonatology, Nanjing Children's Hospital of Nanjing Medical University, Nanjing 210008, P.R. China

\begin{abstract}
Objectives: To examine the protective effects that feeding with expressed maternal milk and donor breast milk compared with formula milk reduces the risk of development of necrotizing enterocolitis in preterm infants.

Data sources: The studies for our systematic review were searched from our library's electronic databases including PUBMED/MEDLINE, SCIENCEDIRECT (1997-2008), EBSCOHOST (1965-2008), EMBASE (1974-2008), OVID (1993-2008) and Cochrane Library.
\end{abstract} trials.

Methods: Systematic review and meta-analysis of randomized controlled trials or quasi-randomized controlled

Results: In our systematic review and meta-analysis only five trials fulfilled the prespecified inclusion criteria. Except for one study, all the rest included studies which were initiated nearly three decades ago. None of the individual trials found any statistically significant difference in the incidence of necrotizing enterocolitis. However, meta-analysis found that preterm infants feeding with donor breast milk was associated with a significantly reduced relative risk of necrotizing enterocolitis. Suspected necrotizing enterocolitis was three times less likely (relative risk $0.31 ; 95 \%$ confidence interval $0.12-0.81 ; p=0.02<0.05$ ) and confirmed necrotizing enterocolitis was four times less likely (relative risk $0.24 ; 95 \%$ confidence interval $0.07-0.76 ; p=0.02<0.05$ ) in premature infants feeding with donor breast milk compared with formula milk given as a sole diet. No data to date was available to be combined in our meta-analysis to compare expressed maternal milk with formula milk given as a sole diet.

Conclusion: Feeding with donor breast milk is associated with a lower risk of necrotizing enterocolitis in preterm infants, but the protective benefits of donor breast milk are described as of borderline effects and the quality of the evidence is limited. Further trials should be focused on the effects of fortified expressed maternal milk and donor breast milk for preventing necrotizing enterocolitis in premature infants.

Keywords: Preterm infants; Necrotizing enterocolitis; Expressed maternal milk; Donor breast milk; Formula milk; Systematic review; Meta-analysis

\section{Introduction}

As advances in neonatology and the modern neonatal intensive care unit (NICU) have improved, much more premature infants could survive after birth. However, necrotizing enterocolitis (NEC) remains a major cause of neonatal morbidity and death. The mortality rate $(15 \%-$ $25 \%)$ for affected infants has not changed appreciably in the past 30 years [1]. Necrotizing enterocolitis is primarily a disease of premature infants; $>90 \%$ of those affected were born prior to 36 weeks gestation [2]. Prematurity is the only risk factor for necrotizing enterocolitis consistently identified in case-control studies. The mechanism of the development of necrotizing enterocolitis is unclear; a leading hypothesis is that the immature intestinal epithelial cells mount an exaggerated inflammatory response to intestinal injury in preterm infants [3].

At present more attention is being focused on the nutritional management of the preterm infants those who are vulnerable to necrotizing enterocolitis. Breast milk is the recommended source of enteral nutrition for all infants including those preterm infants [4]. Compared with formula feeding, a putative benefit of breast milk for feeding preterm infants is that the delivery of immunologic factors to the immature gut mucosa may decrease the risk of necrotizing enterocolitis. However, for some reason mothers those who delivered preterm may be unable to provide directly breast feeding or sufficient breast milk for their premature infants. When directly breast feeding is not available, the preferred alternative is expressed maternal milk (EMM) or donor breast milk (DBM) $[5,6]$.

A theoretical concern is that feeding preterm with expressed maternal milk and donor breast milk do not completely equal to directly breast feeding. Storage and processing of expressed maternal milk and donor breast milk alters some of the immunologic and nutritional properties [7]. Early clinical study suggested that feeding with donor breast milk is associated with lower growth rates in the preterm infants during the short postnatal term [8]. However, it is unclear whether the decrease of non-nutrient components in expressed maternal milk and donor breast milk during storage and processing may confer immunoprotective benefits. The objective of our systematic review and meta-analysis is to determine if enteral feeding with expressed maternal milk and donor breast milk compared with formula milk reduces the incidence of necrotizing enterocolitis in preterm infants.

*Corresponding author: Dr. Xiao-Ming Ben, Department of Neonatology, Nanjing Children's Hospital of Nanjing Medical University, 72 Guangzhou Road, Nanjing 210008, P.R. China, Tel: +86-18951769501; Fax: +86-25-83304239; E-mail: benxm@163.com

Received November 20, 2011; Accepted March 28, 2012; Published March 30 2012

Citation: Ben X, Chen R, Feng Z, Chen T, Zhang J (2012) The Benefits of Expressed Maternal Milk and Donor Breast Milk for Preventing Necrotizing Enterocolitis in Preterm Infants: Systematic Review and Meta- Analysis. J Nutr Disorders Ther 2:110. doi:10.4172/2161-0509.1000110

Copyright: ( 2012 Ben X, et al. This is an open-access article distributed under the terms of the Creative Commons Attribution License, which permits unrestricted use, distribution, and reproduction in any medium, provided the original author and source are credited. 


\section{Methods}

\section{Search strategy}

The standard search strategy of our systematic review included electronic search and manual search. Electronic search was carried out in our library's databases including PUBMED/MEDLINE, SCIENCEDIRECT (1997-2008), EBSCOHOST (1965-2008), EMBASE (1974-2008), OVID (1993-2008) and Cochrane Library. There was no language restriction. As supplement, manual search had also been undertaken; references in studies identified as relevant, and in previous reviews and standard textbooks of neonatal medicine and nutrition were examined.

\section{Selection}

In this systematic we selected studies on the basis of study design, participant, intervention and outcome. Expressed maternal milk had to be collected from the own mother of each preterm infant. Donor breast milk had to be donated from someone other than the infant's mother and it had to be pasteurized or purchased from human milk bank. The studies which had severe methodological faults would be excluded; if the data was equivocal it would be excluded before we got the clarification from its authors. The followings were details for the inclusion criteria:

Study design: Randomized controlled trials (RCTs) or quasi-RCTs.

Participant: Preterm infants $<37$ weeks gestation.

Intervention: Donor breast milk versus formula milk given as a sole diet.

Expressed maternal milk versus formula milk was given as a sole diet. Donor breast milk versus formula milk given as the supplement diet to expressed maternal milk.

Outcome: Suspected NEC (included confirmed NEC and the cases reported by individual trial only without confirmed evidence).

Confirmed NEC (the cases which were confirmed by radiology showing gas in the portal venous system or free air in the abdomen or were confirmed at surgery or autopsy).

\section{Validity assessment}

We assessed the methodological quality of the included randomized controlled trials. Quality of the trials was evaluated in terms of allocation concealment, blinding of parents or carers and assessors to intervention, and completeness of assessment in all randomized subjects. Additional information was requested from the authors of each trial to clarify methodology and results as necessary. Taken clinical heterogeneity into consideration, we also assessed the baseline variables and confounding factors of the included studies.

\section{Data abstraction}

The title and abstract of studies identified via our search strategy were screened by two independent reviewers. The full text of each study which potentially met the inclusion criteria was critically reviewed by both two reviewers. Then the decision to include or exclude a specific article was made by consensus of the two reviewers. If the two reviewers could not get consensus on one article, we would turn to the third reviewer.

\section{Quantitative data synthesis}

Data were separately extracted and summarized into evidence tables by each reviewer, compared data, and resolved differences by consensus. If there were sufficient data and no evidence of significant heterogeneity $(p>0.10)$, meta-analysis would be performed by a fixed-effects model. If there was evidence of significant heterogeneity $(\mathrm{p} \leq 0.10)$ or no sufficient data was available, meta-analysis would be switched to a random-effects model or non-quantitative systematic review would be performed. Effects were expressed as relative risk (RR) and $95 \%$ confidence interval (CI) and risk difference (RD) and 95\% CI for a categorical data, $\mathrm{p}<0.05$ was considered statistically significant. Review Manager (RevMan) (version 5.0.17-A free software used for preparing and maintaining Cochrane reviews downloaded from http://www.cc-ims.net/RevMan/) was used in analysis of data. Our systematic review was conducted and reported in accordance with the guidelines set out in the Cochrane Handbook for Systematic Review of Interventions (version 5.0.1) [9] and the Quality of Reporting of Metaanalyses (QUOROM) statement [10].

\section{Results}

Figure 1 is the QUOROM statement flow diagram of this systematic review. After the first round of screening, thirteen potentially relevant full texts were identified by two independent reviewers. Then those full texts were critically reviewed in accordance with the above inclusion criteria, eight articles [11-18] (seven [11-17] due to no relevant clinical outcome, one [18] due to methodological fault) were excluded on consensus of the two reviewers. At last only five randomized controlled trials were included in our systematic review and meta-analysis.

\section{Description of studies}

The summary of characteristics of the five included trials is shown in the Table 1. There were 862 preterm infants had been enrolled in our systematic review. Four studies [19-22] of these included trials compared donor breast milk with formula milk given as a sole diet for preterm infants. Two studies $[22,23]$ compared donor breast milk with formula milk given as a supplement diet to expressed maternal milk. No data to date was available which compared expressed maternal milk with formula milk given as a sole diet for preterm infants.

Table 2 provides assessments of methodological quality of included trials. Except for one study [21] which was quasi-RCT, all the rest of studies are RCTs with allocation concealment. All the studies had not taken blinding of intervention and none could specify blinding of outcome. Complete follow up was performed in all included studies.

\section{Findings of the included studies}

Effects on suspected NEC: None of the included individual studies

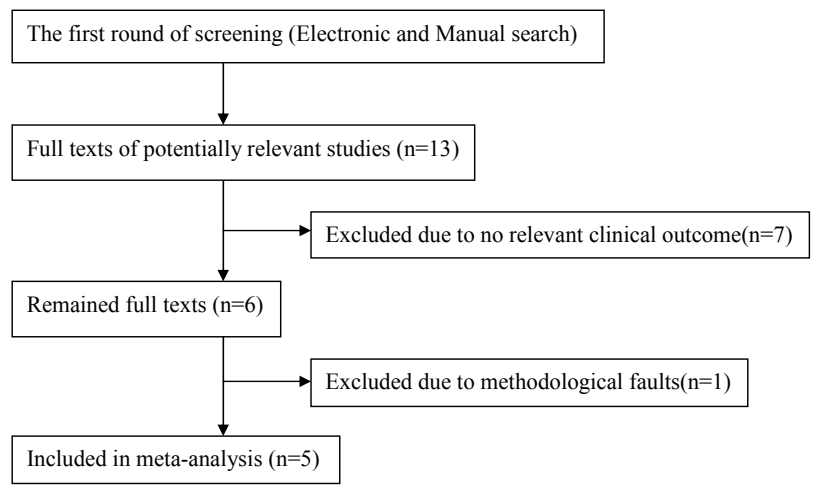

Figure 1: Flow diagram of search and selection of included trials. 
Citation: Ben X, Chen R, Feng Z, Chen T, Zhang J (2012) The Benefits of Expressed Maternal Milk and Donor Breast Milk for Preventing Necrotizing Enterocolitis in Preterm Infants: Systematic Review and Meta- Analysis. J Nutr Disorders Ther 2:110. doi:10.4172/2161-0509.1000110

Page 3 of 6

\begin{tabular}{|c|c|c|c|}
\hline Included Study & Participant & Intervention & Outcome \\
\hline Gross 1983 & $\begin{array}{l}67 \text { preterm infants } \\
\text { Gestation } 27-33 \text { weeks } \\
\text { Birth weight }<1600 \mathrm{~g} \text {. }\end{array}$ & DBM $(n=41)$ vs TFM $(n=26)$ given as sole diet & $\begin{array}{l}\text { Suspected NEC: DBM(1/41) ; TFM(3/26) } \\
\text { Confirmed NEC: } \operatorname{DBM}(1 / 41) ; \operatorname{TFM}(3 / 26)\end{array}$ \\
\hline Tyson 1983 & $\begin{array}{l}81 \text { preterm infants } \\
\text { Birth weight }<1500 \mathrm{~g}\end{array}$ & DBM $(n=37)$ vs PFM $(n=44)$ given as sole diet & $\begin{array}{l}\text { Suspected NEC: DBM(0/37); PFM(2/44) } \\
\text { Confirmed NEC: DBM(0/37); PFM(1/44) }\end{array}$ \\
\hline Cooper 1984 & $\begin{array}{l}39 \text { preterm infants } \\
\text { Gestation< }<36 \text { weeks } \\
\text { Birth weight } 1200-1500 \mathrm{~g}\end{array}$ & DBM $(n=24)$ vs PFM $(n=15)$ given as sole diet & $\begin{array}{l}\text { Suspected NEC: DBM(1/24) ; PFM(3/15) } \\
\text { Confirmed NEC: DBM(1/24); PFM(3/15) }\end{array}$ \\
\hline Lucas 1990A & $\begin{array}{l}159 \text { preterm infants } \\
\text { Birth weight }<1850 \mathrm{~g} .\end{array}$ & DBM $(n=83)$ vs PFM $(n=76)$ given as sole diet & $\begin{array}{l}\text { Suspected NEC: DBM(3/83) ; PFM(6/76) } \\
\text { Confirmed NEC: DBM }(1 / 83) ; \operatorname{PFM}(4 / 76)\end{array}$ \\
\hline Lucas 1990B & $\begin{array}{l}343 \text { preterm infants } \\
\text { Birth weight }<1850 \mathrm{~g} .\end{array}$ & DBM $(n=170)$ vs PFM $(n=173)$ given as the supplement diet for EMM & $\begin{array}{l}\text { Suspected NEC: DBM(8/170) ;PFM(6/173) } \\
\text { Confirmed NEC: DBM(2/170) ;PFM(5/173) }\end{array}$ \\
\hline Schanler 2005 & $\begin{array}{l}173 \text { preterm infants } \\
\text { Gestation } 23-29 \text { weeks }\end{array}$ & DBM $(n=92)$ vs PFM ( $n=81)$ given as the supplement diet for EMM & $\begin{array}{l}\text { Suspected NEC: DBM(5/92); PFM(10/81) } \\
\text { Confirmed NEC: DBM(5/92); PFM(10/81) }\end{array}$ \\
\hline
\end{tabular}

Note: Lucas 1990 A and Lucas $1990 B$ were parallel studies in one trial. DBM: donor breast milk; TFM: term formula milk; PFM: preterm formula milk; EMM: expressed maternal milk; NEC: necrotizing enterocolitis.

Table 1: Characteristics of the included studies.

\begin{tabular}{lllll}
\hline Included Study & Study Design & Allocation Concealment & Blinding of Intervention & Blinding of Outcome \\
\hline $\begin{array}{l}\text { Gross } \\
1983\end{array}$ & RCT & Yes & No & Unclear \\
$\begin{array}{l}\text { Tyson } \\
1983\end{array}$ & RCT & Yes & No & Yes \\
Cooper 1984 & quasi-RCT & Unclear & No & Ynclear \\
$\begin{array}{l}\text { Lucas } \\
1990 \text { A }\end{array}$ & RCT & Yes & No & Unclear \\
$\begin{array}{l}\text { Lucas } \\
1990 \text { B }\end{array}$ & RCT & Yes & No & Unclear \\
Schanler 2005 & RCT & Yes & Yes & Unclear \\
\hline
\end{tabular}

Note: Lucas 1990A and Lucas 1990B were parallel studies in one trial. RCT: randomized controlled trial.

Table 2: Methodological quality of the included studies.

either in the sole diet comparison or supplement diet comparison found any statistically significant difference in the incidence of Suspected NEC. When the data from the included trials were combined in a meta-analysis, a statistically significant difference (RR 0.54; $95 \% \mathrm{CI}$ 0.31-0.95; $\mathrm{p}=0.03<0.05)$ was shown in the incidence of Suspected NEC (Figure 2). In the subgroup meta-analysis of the sole diet comparison (Figure 3) we also found a statistically significant difference (RR 0.31 ; 95\% CI $0.12-0.81 ; \mathrm{p}=0.02<0.05)$. However, there was no significant difference (RR 0.77; 95\% CI 0.38-1.55; $\mathrm{p}=0.46>0.05$ ) in the subgroup meta-analysis of the supplement diet comparison (Figure 4).

Effects on confirmed NEC: No statistically significant difference in the incidence of Confirmed NEC was shown in all the included individual studies. However, a significant difference (RR 0.34; 95\% CI 01.7-0.68; $\mathrm{p}=0.002<0.05)$ was shown when the individual data were combined in meta-analysis (Figure 5). As the effects on Suspected NEC, a significant difference (RR 0.24; 95\% CI 0.07-0.76; $p=0.02<0.05$ ) was seen in the subgroup meta-analysis of the sole diet comparison but no significant difference (RR 0.43; 95\% CI 0.18-1.03; $\mathrm{p}=0.06>0.05$ ) in the supplement diet comparison (Figure 6,7).

\section{Sensitivity analysis}

Sensitivity analysis is an approach to test how robust the results of our systematic review are relative to key decisions and assumptions that were made in the process of conducting the review. Preterm infants enrolled in the formula milk group were fed with term formula milk in one included study [19]. Term formula milk is different with preterm formula milk which was used in other included studies. To eliminate this potential confounding factor, the data abstracted from the studies in which preterm infants were fed with preterm formula milk, were combined in sensitivity analysis, and we found a statistically significant difference (RD -0.05; 95\% CI -0.10-0.00; $\mathrm{p}=0.03<0.05$ ) (Figure 8 ).

\section{Discussion}

In order to get the appropriate conclusion from our systematic review and meta-analysis, the limitations should be carefully considered before we discuss its results. First of all, there were only five included trials in our meta-analysis and no data was available to compare expressed maternal milk versus preterm formula milk given as a sole diet, therefore a noteworthy publication bias examined by 'funnel-plot' was inevitable. For another, except for one study [23], the rest included studies which were started nearly three decades ago, and in the past 30 years the composition of the formula milk and feeding practices of preterm infants had changed greatly. Still another, there were several methodological faults in terms of randomization, allocation concealment, blinding of intervention and outcome, and a degree of clinical heterogeneity. These limitations would be considered carefully in our following discussion.

Based on the available data of the included studies, our metaanalysis suggests that donor breast milk has a degree of protective effects for preventing necrotizing enterocolitis in preterm infants. Although none of the included studies individually found any significant difference, the significant differences were shown in our meta-analysis. Furthermore, the relative risk estimates and the risk difference estimate are both statistically significant, the results of sensitivity analysis are consistent with the overall effects shown in 


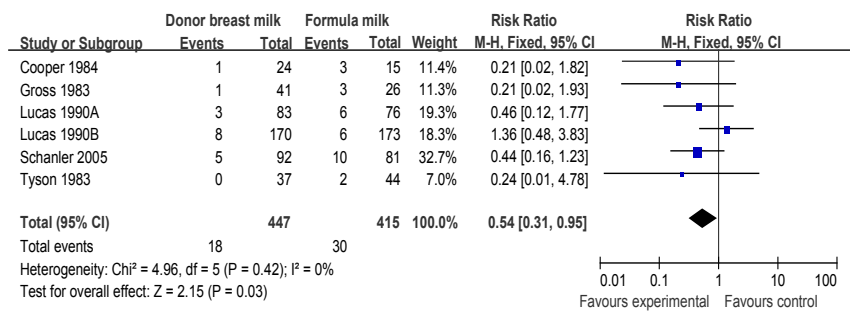

Note: Relative risk of Suspected NEC in preterm infants fed with donor breas milk vs formula milk (RR: $0.54 ; 95 \% \mathrm{Cl}$ : $0.31-0.95 ; p=0.03$ ), the overall effect favors donor breast milk.

Figure 2: Relative risk of Suspected NEC in preterm infants fed with donor breast milk vs formula milk.

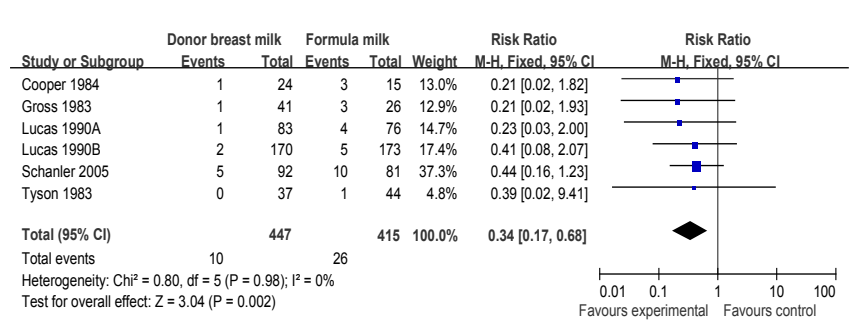

Note: Relative risk of Confirmed NEC in preterm infants fed with donor breas milk vs formula milk (RR: $0.34 ; 95 \% \mathrm{Cl}: 0.17-0.68 ; p=0.002$ ), the overall effect favors donor breast milk.

Figure 3: Relative risk of Confirmed NEC in preterm infants fed with donor breast milk vs formula milk.

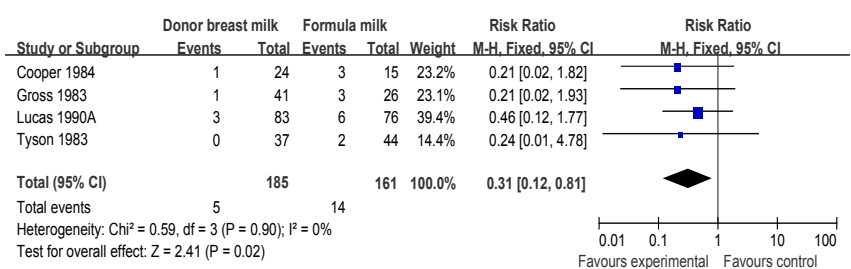

Note: Relative risk of Suspected NEC in preterm infants fed with donor breas milk vs formula milk given as a sole diet (RR: $0.31 ; 95 \% \mathrm{Cl}: 0.12-0.81 ; p=0.02$ ) the overall effect favors donor breast milk.

Figure 4: Relative risk of Suspected NEC in preterm infants fed with donor breast milk vs formula milk given as a sole diet.

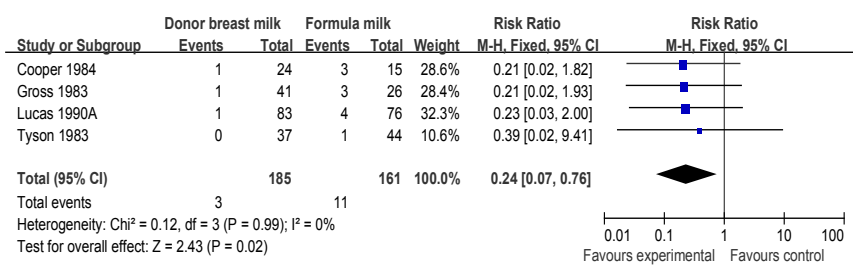

Note: Relative risk of Confirmed NEC in preterm infants fed with donor breast milk vs formula milk given as a sole diet (RR: $0.24 ; 95 \% \mathrm{Cl}$ : $0.07-0.76 ; p=0.02$ ), the overall effect favors donor breast milk.

Figure 5: Relative risk of Confirmed NEC in preterm infants fed with donor breast milk vs formula milk given as a sole diet.

meta-analysis. Suspected NEC is three times less likely and Confirmed NEC is four times less likely in premature infants feeding with donor breast milk compared with formula milk given as a sole diet. Subgroup analysis was performed in this review due to clinical heterogeneity. In subgroup analysis we compared donor breast milk with preterm formula milk given as a supplement diet to expressed maternal milk for preterm infants, and no significant difference was found. No data was available to compared expressed maternal milk with preterm formula milk or donor breast milk. Only one individual study [23] reported the incidence of necrotizing enterocolitis in preterm infants fed with expressed maternal milk given as a sole diet, and no benefit was found. The potential benefits of expressed maternal milk for preventing necrotizing enterocolitis may be estimated in the future systematic review when sufficient data are available.

Up to now the pathophysiology of necrotizing enterocolitis remains poorly delineated, evidence supports prematurity is an important risk factor and the beneficial effects of breast milk reduce the risk for necrotizing enterocolitis in premature infants [24]. Feeding preterm infants with breast milk would deliver an amount of immunoprotective factors and growth factors to the immature gut mucosa that may decrease the risk of necrotizing enterocolitis. According to basic research, one of the constituents of breast milk that may prove to be therapeutic is epidermal growth factor (EGF), a trophic substance for intestinal growth [25]. A preliminary study of epidermal growth factor

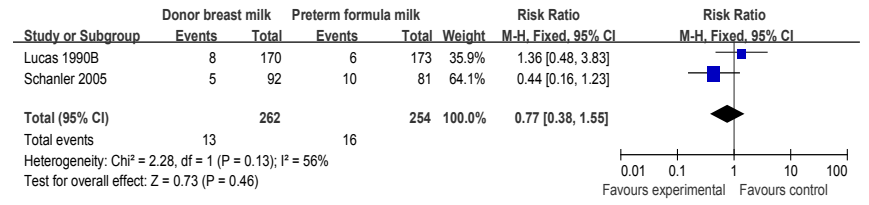

Note: Relative risk of Suspected NEC in preterm infants fed with donor breast milk vs formula milk given as the supplement diet to expressed maternal milk (RR: $0.77 ; 95 \% \mathrm{Cl}: 0.38-1.55 ; \mathrm{p}=0.46$ ), no statistically significant difference.

Figure 6: Relative risk of Suspected NEC in preterm infants fed with donor breast milk vs formula milk given as the supplement diet to expressed maternal milk.

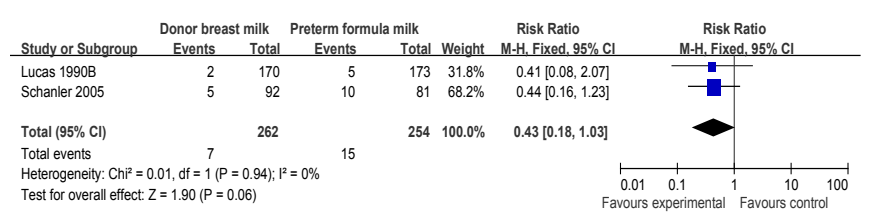

Note: Relative risk of Confirmed NEC in preterm infants fed with donor breast milk vs formula milk given as the supplement diet to expressed maternal milk (RR: $0.43 ; 95 \% \mathrm{Cl}: 0.18-1.03 ; p=0.06$ ), no statistically significant difference.

Figure 7: Relative risk of Confirmed NEC in preterm infants fed with donor breast milk vs formula milk given as the supplement diet to expressed maternal milk.

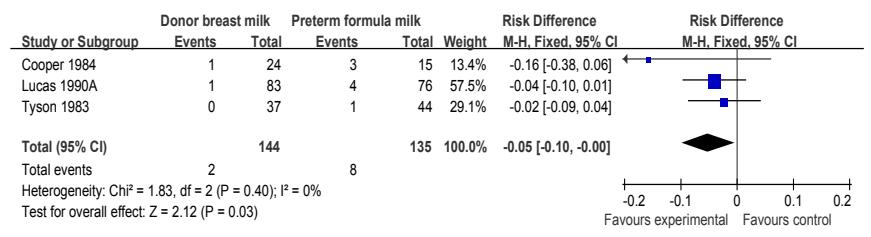

Note: Risk difference of Confirmed NEC in preterm infants fed with donor breast milk vs preterm formula milk given as a sole diet (RD: $-0.05 ; 95 \% \mathrm{Cl}$ : $-0.10-0.00 ; p=0.03$ ), the overall effect favors donor breast milk.

Figure 8: Risk difference of Confirmed NEC in preterm infants fed with donor breast milk vs preterm formula milk given as a sole diet. 
in neonates diagnosed with necrotizing enterocolitis has shown that epidermal growth factor promotes the repair of intestinal epithelium, and supplementation of epidermal growth factor in animal models has decreased the incidence of necrotizing enterocolitis [26]. Donor breast milk is generally obtained from women who deliver term infants later in their lactation and a loss of the immunologic and nutritional properties during storage and processing in the human milk bank, so the milk has a lower content of protein and host defence protein [7,27]. Although the immunoprotective factors delivered to preterm infants is a small amount by donor breast milk, it may account for the borderline protective effect for preventing necrotizing enterocolitis in our metaanalysis.

Expressed maternal milk is obtained from their own mothers for preterm infants, so it is a form of preterm milk. The results of studies compared preterm milk with term milk suggested that there were much more immunologic and nutritional properties in preterm milk [28]. Theoretically expressed maternal milk is more appropriate than donor breast milk for feeding premature infants, but why no significant difference was found in meta-analysis when donor breast milk versus preterm formula milk were given as a supplement diet to expressed maternal milk? Recently one study enrolled in 1272 preterm infants was performed to determine the association between breast milk intake and risk of necrotizing enterocolitis or death among infants 401 to $1000 \mathrm{~g}$ birth weight [17]. This study suggested that a doserelated association of breast milk feeding with a reduction of risk of necrotizing enterocolitis or death after the first two weeks of life among extremely low birth weight infants [17]. These findings may help us elucidate the puzzled results of meta-analysis. When donor breast milk (contains a small amount of immunoprotective factors) versus preterm formula milk (contains no immunoprotective factor) were given as a supplement diet to expressed maternal milk (contains much more immunoprotective factors), the difference of the dose-related effects between the two groups may become relatively less significant.

Are these findings in our systematic review of clinical significance? In meta-analysis with several limitations, we found that donor breast milk was associated with a borderline effect for preventing necrotizing enterocolitis in premature infants. However, the number needed to treat $(\mathrm{NNT}=1 / \mathrm{RD})$ was 20 (that meant one case of Confirmed NEC averted if 20 preterm infants received donor breast milk). Additional, donor breast milk given as a sole diet for preterm infants is associated with slower growth at least in the early postnatal period, the longterm effect is unclear $[8,29]$. The nutrient concentrations in expressed maternal milk and donor breast milk may be inadequate for preterm infants, who have increased nutritional requirements [30]. The nutrient deficits that arise from feeding unfortified donor breast milk may be corrected with nutrient supplementation, and in many clinical guidelines fortifiers were recommended when necessary $[5,6]$. However, a theoretical concern with human milk fortification is that the added nutrients may affect the intrinsic host defense system of the milk, thereby may increase the risk of development of necrotizing enterocolitis in preterm infants. Although a meta-analysis comparing infants fed unfortified and fortified human milk did not identify any significant difference in necrotizing enterocolitis [31], further research in the safety of human milk fortification is still needed.

In summary, donor breast milk given as a sole diet is associated with lower risk of the development of necrotizing enterocolitis in preterm infants. The clinical applicability of these findings should be considered with caution due to the limitations of our meta-analysis itself and the potential adverse effects on growth found in other studies. On our systematic review itself, the inspiration significance is the most important rather than its findings' clinical applicability. Consequently, further high quality randomized controlled trials are still needed, and should be focused on the protective effects of fortified expressed maternal milk and donor breast milk for preterm infants, especially the dose-related effects for preventing necrotizing enterocolitis in the extremely low birth weight infants.

\section{References}

1. Henry MC, Moss RL (2009) Necrotizing enterocolitis. Annu Rev Med 60: 111 124

2. Guthrie SO, Gordon PV, Thomas V, Thorp JA, Peabody J, et al. (2003) Necrotizing enterocolitis among neonates in the United States. J Perinatol 23 278-285.

3. Lin PW, Nasr TR, Stoll BJ (2008) Necrotizing enterocolitis: recent scientific advances in pathophysiology and prevention. Semin Perinatol 32: 70-82.

4. McGuire W, Henderson G, Fowlie PW (2004) Feeding the preterm infant. BMJ 329: $1227-1230$

5. (1997) Breastfeeding and the use of human milk. American Academy of Pediatrics. Work Group on Breastfeeding. Pediatrics 100: 1035-1039.

6. Gartner LM, Morton J, Lawrence RA, Naylor AJ, O'Hare D, et al. (2005) Breastfeeding and the use of human milk. Pediatrics 115: 496-506.

7. Wight NE (2001) Donor human milk for preterm infants. J Perinatol 21: 249 254

8. Lucas A, Gore SM, Cole TJ, Bamford MF, Dossetor JF, et al. (1984) Multicentre trial on feeding low birthweight infants: effects of diet on early growth. Arch Dis Child 59: 722-730.

9. Higgins JPT, Green S (editors) (2011) Cochrane Handbook for Systematic Reviews of Interventions Version 5.1.0 [updated March 2011]. The Cochrane Collaboration.

10. Moher D, Cook DJ, Eastwook S, Olkin I, Rennie D, et al. (1999) Improving the quality of reports of meta-analyses of randomised controlled trials: the QUOROM statement. Quality of Reporting of Meta-analyses. Lancet 354 1896-1900.

11. Davies DP (1977) Adequacy of expressed breast milk for early growth of preterm infants. Arch Dis Child 52: 296-301.

12. Schultz K, Soltész G, Mestyán J (1980) The metabolic consequences of human milk and formula feeding in premature infants. Acta Paediatr Scand 69: 647652

13. Narayanan I, Prakash K, Gujral VV (1981) The value of human milk in the prevention of infection in the high-risk low-birth-weight infant. J Pediatr 99: 496498.

14. Järvenpää AL, Räihä NC, Rassin DK, Gaull GE (1983) Feeding the low-birthweight infant: I. Taurine and cholesterol supplementation of formula does not affect growth and metabolism. Pediatrics 71: 171-178.

15. Putet G, Senterre J, Rigo J, Salle B (1984) Nutrient balance, energy utilization, and composition of weight gain in very-low-birth-weight infants fed pooled human milk or a preterm formula. J Pediatr 105: 79-85

16. O'Connor DL, Jacobs J, Hall R, Adamkin D, Auestad N, et al. (2003) Growth and development of premature infants fed predominantly human milk, predominantly premature infant formula, or a combination of human milk and premature formula. J Pediatr Gastroenterol Nutr 37: 437-446.

17. Meinzen-Derr J, Poindexter B, Wrage L, Morrow AL, Stoll B, et al. (2009) Role of human milk in extremely low birth weight infants' risk of necrotizing enterocolitis or death. J Perinatol 29: 57-62.

18. Svenningsen NW, Lindroth M, Lindquist B (1982) Growth in relation to protein intake of low birth weight infants. Early Hum Dev 6: 47-58.

19. Gross SJ (1983) Growth and biochemical response of preterm infants fed human milk or modified infant formula. N Engl J Med 308: 237-241.

20. Tyson JE, Lasky RE, Mize CE, Richards CJ, Blair-Smith N, et al. (1983) Growth, metabolic response, and development in very-low-birth-weight infants fed banked human milk or enriched formula. I. Neonatal findings. J Pediatr 103.95-104. 
Citation: Ben X, Chen R, Feng Z, Chen T, Zhang J (2012) The Benefits of Expressed Maternal Milk and Donor Breast Milk for Preventing Necrotizing Enterocolitis in Preterm Infants: Systematic Review and Meta- Analysis. J Nutr Disorders Ther 2:110. doi:10.4172/2161-0509.1000110

21. Cooper PA, Rothberg AD, Pettifor JM, Bolton KD, Devenhuis S (1984) Growth and biochemical response of premature infants fed pooled preterm milk or special formula. J Pediatr Gastroenterol Nutr 3: 749-754.

22. Lucas A, Cole TJ (1990) Breast milk and neonatal necrotising enterocolitis. Lancet 336: 1519-1523.

23. Schanler RJ, Lau C, Hurst NM, Smith EO (2005) Randomized trial of donor human milk versus preterm formula as substitutes for mothers' own milk in the feeding of extremely premature infants. Pediatrics 116: 400-406.

24. Schanler RJ, Shulman RJ, Lau C (1999) Feeding strategies for premature infants: beneficial outcomes of feeding fortified human milk versus preterm formula. Pediatrics 103: 1150-1157.

25. Warner BW, Warner BB (2005) Role of epidermal growth factor in the pathogenesis of neonatal necrotizing enterocolitis. Semin Pediatr Surg 14: $175-180$.
26. Nair RR, Warner BB, Warner BW (2008) Role of epidermal growth factor and other growth factors in the prevention of necrotizing enterocolitis. Semin Perinatol 32: 107-113.

27. Heiman H, Schanler RJ (2006) Benefits of maternal and donor human milk for premature infants. Early Hum Dev 82: 781-787.

28. Suzuki S, Lucas A, Lucas PJ, et al. (1983) Immunoglobulin concentrations and bacterial antibody titres in breast milk from mothers of 'preterm' and 'term' infants. Acta Paediatr Scand 72: 671-677.

29. Boyd CA, Quigley MA, Brocklehurst $P$ (2007) Donor breast milk versus infant formula for preterm infants: systematic review and meta-analysis. Arch Dis Child Fetal Neonatal Ed 92: F169-F175.

30. Lawrence RA, Lawrence RM (1998) Breastfeeding. A guide for the medical profession, fifth edition.

31. Kuschel CA, Harding JE (2005) Multicomponent fortified human milk for promoting growth in preterm infants. The Cochrane Library 1-36. 